\title{
Born's rule from measurements of classical signals by threshold detectors which are properly calibrated
}

\author{
Andrei Khrennikov \\ International Center for Mathematical Modelling \\ in Physics and Cognitive Sciences \\ Linnaeus University, Växjö, S-35195, Sweden \\ Andrei.Khrennikov@lnu.se \\ tel. $46-470708790$
}

November 1, 2018

\begin{abstract}
The very old problem of the statistical content of quantum mechanics (QM) is studied in a novel framework. The Born's rule (one of the basic postulates of QM) is derived from theory of classical random signals. We present a measurement scheme which transforms continuous signals into discrete clicks and reproduces the Born's rule. This is the sheme of threshold type detection. Calibration of detectors plays a crucial role.
\end{abstract}

\section{Introduction}

In this paper we stress the role of detectors and more generally the detection procedure in the debate on completeness of QM. This debate was originated in the famous paper of Einstein, Podolsky, and Rosen [1] in 1933 and it still attracts a lot of attention of experts in quantum foundations and quantum information (including researchers working on applied issues and experimentalists, especially in quantum optics), 
see, e.g., 2]-9]. Surprisingly the role of detectors and the detection procedure in the problem of hidden variables has not yet been properly analysed. Typically, one points to detectors' inefficiency loophole in experimental tests of Bell's inequality, e.g., [10]-[12] or generally unfair sampling [13] but not more. And in such formal treatement it is considered as merely a technological problem which cannot be reposible for nonclassicality of statitsical data from quantum experiments 1 However, more detailed study, e.g., [14, of what happens in real laboratories, e.g., [15-18, demonstrated that the process of detection and real experimental routine cannot be regarded as simply technicalities. It seems that they play an important role in production of nonclassical statistics (and the author guesses - the crucial role).

In this paper we show that it is really the case: the basic postulate of QM, Born's rule, can be derived by taking into account the presence of detection threshold and calibration of detectors. The latter is crucial to exclude double clicks in "single particle" detection, cf. the experiment of Grangier et al. [19], 20]. In fact, all existing detectors photomultipliers tubes - PMTs, avalanche photodiodes - APDs, visible light counters - VLPCs, supeconducting bolometers - SPD are of such a type and all detectors have to be calibrated, see, e.g., [21]-23].

The classical ("prequantum") model under consideration is of the wave type: classical fields are selected as hidden variables. (So, the state space of hidden variables, the $L_{2}\left(\mathbf{R}^{3}\right)$-space, is infinite dimensional.) This is prequantum classical statisstical field theory (PCSFT) which was recently developed by the author and collaborators [24][27.

We state again that the field-type prequantum models are quite popular among those who question the completeness of QM, e.g., [28][46]. At the very beginning of the quantum theory, Schrödinger was sure that he simply discovered a new kind of wave mechanics 30. He interpreted the wave function of electron $\psi(x)$ (not normalized!) as the density of electron's charge. Hence, the quantity

$$
q(V)=e \int_{V}|\psi(x)|^{2} d x
$$

\footnotetext{
${ }^{1}$ For example, Alain Aspect told to the author that he does not consider the problem of detectors' inefficiency as a problem of the fundamental value for quantum foundations (private communication, conference "Foundations of Probability and Physics-4", Växjö 2007). Therefore he was not sure that the quantum community has to put resources to close this loophole.
} 
is the electron charge in the domain $V$. Here $e$ is the elementary electron charge. Shrödinger derived his equation starting with De Broglie's idea to associate a wave with any quantum particle 28]. Later De Brogle developed his Double Solution Theory [29] in which the wave function was interpreted as a physical wave. (Particles appear as singularities in those waves.) His approach was essentially elaborated by Bohm - to the pilot wave prequantum model, e.g., [37. We remark that our own approach PCSFT is closer to first attempts of Schrödinger to proceed in a purely wave framework, i.e., to consider e.g. the wave of electron charge, not electron-particle. Oppoiste to early Schrödinger and our approach, Bohmian mechanics is not aimed towards a purely wave model of prequantum reality. PCSFT is close to the idea of Einstein to create a purely field model of physical reality, see, e.g., Einstein and Infeld 32 . Among other wave-like prequantum models, we also mention stochastic electrodynamics and semiclassical approach [33]-46].

In this paper we show that PCSFT, a model of classical field type, can be completed by detection theory which produces Born's rule for probabilities of clicks of detectors. This is an important improvement of PCSFT. In its previous version [24]-227, it reproduced quantum averages and correlations, but as averages and correlations of (continuous) intensities of classical signals. Transition from signal inetensity to probability of discrete clicks is a real step towards quantum-like representation of the classical field theory. We discuss this issue more carefully.

Consider the classical electromagnetic field $(E(x), B(x))$, where $E(x)$ and $B(x)$ are electric and magnetic fields, respectively. To make the analogy with QM closer, we use Riemann-Silberstein representation:

$$
\psi(x)=E(x)+i B(x) .
$$

(We remark that this complex function is not $L_{2}$-normalized.) Then the energy of the field concentrated in the domain $V$ is given by

$$
\mathcal{E}(V)=\int_{V}|\psi(x)|^{2} d x
$$

cf. (11). Of course, the formula (2) as well as (11) remind very much Born's rule

$$
P(x \in V)=\int_{V}|\Psi(x)|^{2} d x,
$$


where we use the symbol $\Psi$ to distinguish the normalized wave function from non-normalized physical field. The first formal difference between (2) and (3) is aforementioned normalization. However, this is a minory problem. Consider the total energy of the calssical electromagnetic field

$$
\mathcal{E}=\|\psi\|^{2}=\int_{\mathbf{R}^{3}}|\psi(x)|^{2} d x,
$$

Then the realative portion of electromagnetic energy concentrated in the domain $V$ is given by

$$
\frac{\mathcal{E}(V)}{\mathcal{E}}=\frac{1}{\|\psi\|^{2}} \int_{V}|\psi(x)|^{2} d x=\int_{V}|\Psi(x)|^{2} d x .
$$

where

$$
\Psi(x)=\frac{\psi(x)}{\|\psi\|}=\frac{E(x)+B(x)}{\sqrt{E^{2}(x)+B^{2}(x)}},
$$

is the "normalized electromagnetic field". Therefore it is natural to guess that Born's rule (3) for probability of detection reflects in some way the rule for relative energy concentration 2 The main problem is that Born's rule (3) is about probability of discrete clicks and the rule (4) is about intensity of continuous signal. In this paper we show that Born's rule (3) can be really derived from (5) and not only for the electromagnetic field.

Besides aforementioned restrictions on the detection procedure, the derivation is based on an important mathematical assumption on prequantum random fields. They have to be ergodic. Ergodicity provides a possibility to reduce ensemble averages to time averages and this is the cornerstone of our derivation.

Some readers might be not so much interested in a new model with hidden variables. In such a case the paper can be considered as a test of boundaries of simulation of quantum statistical data with the aid of classical signal theory.

Therefore we do not start directly with the prequantum model PCSFT. We start with classical theory of random signals, section 2 , cf. [47, 48]. Then we derive Born's rule for threshold type detectors, section 3 and represent the calibration procedure in this framework, section 4. (We emphasize that in our model the probability of detection is not changed by calibration.) In section 5 we demonstrate

\footnotetext{
${ }^{2}$ According to quantum folklore, Max Born invented his rule starting with the Schrödinger's rule (11) for the electric charge density.
} 
that one can exclude double clicks in experiments with "single particle detection" - as in the two slit experiment, in context such that two detectors are placed behind two slits (and single photon source). Here the proper calibration plays a crucial role. Finally, in section 6 we present our model with hidden variables PCSFT and then couple it to the detection scheme elaborated in sections 3, 4, 5,

We point to coupling with the operational approach to QM, cf., e.g., [49]-52. In fact, we present the operational approach to measurement of classical random signals. This approach reprodcues quantum probabilities. (Of course, we are well aware that the majority of the operational community stays firmly on the Copenhagen's position, i.e., that $\mathrm{QM}$ is complete.)

We also remark that recently the question of validity of Born's rule has attracted a lot of attention. We can mention the experimental test [53, [54] of Sorkin's model (see, e.g., [53] for brief presentation) with violation of Born's rule for triple-slit experiment and ideas on quantum supercomputing based on quantum-like models violating Born's rule [55]. By PCSFT (completed with measurement theory developed in this paper) validity of Born's rule (3) in QM is a consequence of the formula (2) (in fact, its "normalized version" (50)), i.e., the fact that in classical signal theory the energy at the point $x \in \mathbf{R}^{3}$ is proportional to squared field

$$
\mathcal{E}(x) \equiv \mathcal{E}_{2}(x)=|\psi(x)|^{2} .
$$

Of course, the assumption that detectors integrate the energy density plays a crucial role. If some kind of detector also were able to integrate nonquadratic field nonlinearities, then Born's rule can be violated (in measurements with detectors of such a type). For example [56], suppose that a detector can also integrate the contribution of the quantity

$$
\mathcal{E}_{4}(x)=|\psi(x)|^{4} .
$$

Hence, totally the quantity

$$
\mathcal{E}_{2,4}(x)=|\psi(x)|^{2}+\alpha|\psi(x)|^{4},
$$

where $\alpha$ is a small parameter, contributes to a click. Then Born's rule has to be violated:

$$
P(x \in V)=\int_{V}|\Psi(x)|^{2} d x+\alpha F\left(|\Psi|^{2},|\Psi|^{4}\right) .
$$


In [56] the addtitional contribution $F\left(|\Psi|^{2},|\Psi|^{4}\right)$ has been calculated. The small parameter $\alpha$ determines the scale of prequantum fluctuations, so if a detector even were able to integrate fourth-order nonlinearity, the corresponding perturbation would be very small.

Thus, by PCSFT-approach to QM Born's rule might be in principle violated. However, this would not have a fundamental value for theory, since such violation would be coupled solely to the detection procedure and not properties of the prequantum random fields.

\section{Classical random signals: ensemble and time representations of averages}

The state space of classical signal theory is the $L_{2}$-space

$$
H=L_{2}\left(\mathbf{R}^{3}\right) .
$$

Elements of $H$ are classical fields $\phi: \mathbf{R}^{3} \rightarrow \mathbf{C}^{n}$. We consider complex valued fields. (For example, for the classical electromagnetic field we use Riemann-Silberstein representation, $\phi(x)=E(x)+i B(x)$.)

A random field (signal) is a field (signal) depending on a random parameter $\omega, \phi(x, \omega)$. In the measure-theoretic framework (Kolmogorov, 1933) it is represented as $H$-valued random variable, $\omega \rightarrow$ $\phi(\omega) \in H$. Its probability distribution is denoted by the symbol $\mu$ on $H$.

Consider functionals of fields,

$$
f: H \rightarrow \mathbf{C}, \phi \rightarrow f(\phi) .
$$

These are physical observables for classical signals. For example, the energy of the classical electromagnetic field is geven by the quadratic functional

$$
f(\phi) \equiv f(E, B)=\int_{\mathbf{R}^{3}}|\phi(x)|^{2} d x=\int_{\mathbf{R}^{3}}\left(E^{2}(x)+B^{2}(x)\right) d x .
$$

The average of an observable can be written as the integral over the space of fields

$$
\langle f\rangle=\int_{H} f(\phi) d \mu(\phi)
$$

To find $\langle f\rangle$, we consider an ensemble (in theory infinite) of realizations of the random field and calculate the average of $f(\phi)$ with respect to 
this ensemble. This measure-theoretic (ensemble) representation is very convenient in theoretical considerations [47, [48. However, in practice we never produce an ensemble of different realizations of a signal. Instead of this, we have a single time dependent realization of a signal, $\phi(s, x)$. It is measured at different instances of time. Finally, we calculate the time average. The latter is given by

$$
\bar{f}=\lim _{\Delta \rightarrow \infty} \frac{1}{\Delta} \int_{0}^{\Delta} f(\phi(s)) d s .
$$

In classical signal theory [47, [48] the ensemble and time averages are coupled by the ergodicity assumption. Under this assumption we obtain that

$$
\bar{f}=\langle f\rangle,
$$

i.e.,

$$
\int_{H} f(\phi) d \mu(\phi)=\lim _{\Delta \rightarrow \infty} \frac{1}{\Delta} \int_{0}^{\Delta} f(\phi(s)) d s \approx \frac{1}{\Delta} \int_{0}^{\Delta} f(\phi(s)) d s,
$$

foe sufficiently large $\Delta$.

In coming consideration we shall operate only with observables given by quadratic functionals of classical signals

$$
\phi \rightarrow f_{A}(\phi)=\langle\widehat{A} \phi, \phi\rangle
$$

where $\widehat{A}$ is a self-adjoint operator. Moreover, to describe a procedure of the position detection we need only functionals of the form

$$
\phi \rightarrow\left|\phi\left(x_{0}\right)\right|^{2}
$$

where $x_{0} \in \mathbf{R}^{3}$ is a fixed point which determines the quadratic functional (later $x_{0}$ will be considered as the position of a detector).

In what follows we consider only random signals with covariance operators of the type

$$
D_{\psi}=|\psi\rangle\langle\psi|
$$

where $\psi \in H$ is arbitrary vector (i.e., it need not be normalized by 1 ).

We remark that we still proceed in the framework of classical signal theory, i.e., without any relation to QM. We simply consider a special class of classical random signals characterized by the form of the covariance operator. For example, we can consider Gaussian beams. For a Gaussian signal (with zero mean value), the form (15) of the 
covariance operator implies that this signal is concentrated on one dimensional subspace of the $L_{2}$-space based on the vector $\psi$. We also state again that for Gaussian signals the covariance operator determines signal uniquely (for the fixed mean value).

For such probability measure $\mu \equiv \mu_{\psi}$,

$$
\left\langle f_{x_{0}}\right\rangle=\int_{H}\left|\phi\left(x_{0}\right)\right|^{2} d \mu_{\psi}(\phi)=\left|\psi\left(x_{0}\right)\right|^{2} .
$$

And under the assumption of ergodicity, we obtain

$$
\left|\psi\left(x_{0}\right)\right|^{2}=\lim _{\Delta \rightarrow \infty} \frac{1}{\Delta} \int_{0}^{\Delta}\left|\phi\left(s, x_{0}\right)\right|^{2} d s \approx \frac{1}{\Delta} \int_{0}^{\Delta}\left|\phi\left(s, x_{0}\right)\right|^{2} d s,
$$

for sufficiently large $\Delta$. Consider the functional

$$
\pi(\phi)=\|\phi\|^{2}=\int_{\mathbf{R}^{3}}|\phi(x)|^{2} d x .
$$

In PCSFT it represents the total energy of a signal. We find its average. In general,

$$
\langle\pi\rangle=\int_{H} \pi(\phi) d \mu(\phi)=\operatorname{TrD}_{\mu} .
$$

In particular, for $\mu=\mu_{\psi}$,

$$
\langle\pi\rangle=\int_{H} \pi(\phi) d \mu_{\psi}(\phi)=\|\psi\|^{2} .
$$

By ergodicity

$$
\langle\pi\rangle=\|\psi\|^{2}=\lim _{\Delta \rightarrow \infty} \frac{1}{\Delta} \int_{0}^{\Delta}\|\phi(s)\|^{2} d s \approx \frac{1}{\Delta} \int_{0}^{\Delta} d s \int_{\mathbf{R}^{3}} d x|\phi(s, x)|^{2},
$$

for sufficiently large $\Delta$.

If, as usual in signal theory, the quantity $|\phi(s, x)|^{2}$ has the physical dimension of the energy density, i.e., energy/volume, then by selecting some unit of time denoted $\gamma$ we can interpret the quantity

$$
\frac{1}{\gamma} \int_{0}^{\Delta}\left|\phi\left(s, x_{0}\right)\right|^{2} d s d V
$$

as the energy which can be collected in the volume $d V$ during the time interval $\Delta$ (from the random signal $\phi(s) \in H$ ). In the same way

$$
\frac{1}{\gamma} \int_{0}^{\Delta} d s \int_{\mathbf{R}^{3}} d x|\phi(s, x)|^{2}
$$

is the total energy which can be collected during the time interval $\Delta$. Its time average can be represented in the form (21). 


\section{Discrete-counts model for detection of classical random signals}

We consider the following model of detector's functioning. Its basic parameter is detection threshold energy $\epsilon \equiv \epsilon_{\text {click }}$. The detector under consideration clicks after it has been collected the energy

$$
E_{\text {collected }} \approx \epsilon .
$$

Such a detector is calibrated to work in accordance with (24). Clicks with energies deviating from $\epsilon$ are discarded. Detectors are calibrated for a class of signals and $\epsilon$ is selected as

$$
\epsilon \approx\langle\pi\rangle=\|\psi\|^{2},
$$

the average energy of a signal.

Select $\gamma$ as e.g. one second. Consider such a detector located in small volume $d V$ around a point $x_{0} \in \mathbf{R}^{3}$. In average it clicks each $\Delta$ seconds, where $\Delta$ is determined form the approximative equality

$$
\frac{1}{\gamma} \int_{0}^{\Delta}\left|\phi\left(s, x_{0}\right)\right|^{2} d s d V \approx \epsilon
$$

or

$$
\frac{\Delta}{\gamma}\left(\frac{1}{\Delta} \int_{0}^{\Delta}\left|\phi\left(s, x_{0}\right)\right|^{2} d s\right) d V \approx\|\psi\|^{2}
$$

or

$$
\frac{\Delta}{\gamma}\left|\psi\left(x_{0}\right)\right|^{2} d V \approx\|\psi\|^{2} .
$$

Let us introduce the normalized function

$$
\Psi(x)=\psi(x) /\|\psi\|,
$$

i.e., $\|\Psi\|^{2}=1$. Then (28) can be rewritten as

$$
\frac{\Delta}{\gamma}\left|\Psi\left(x_{0}\right)\right|^{2} d V \approx 1
$$

Thus at the point $x_{0}$ such a detector clicks (in average) with the frequency

$$
\lambda\left(x_{0}\right)=\frac{\gamma}{\Delta} \approx\left|\Psi\left(x_{0}\right)\right|^{2} d V .
$$

This frequency of clicks coincides with the probability of detection at the point $x_{0}$. 
Consider a large interval of time, say $T$. The number of clicks at $x_{0}$ during this interval is given by

$$
n_{T}\left(x_{0}\right)=\frac{T}{\Delta} \approx \frac{1}{\gamma}\left|\Psi\left(x_{0}\right)\right|^{2} d V .
$$

The same formula is valid for any point $x \in \mathbf{R}^{3}$. Hence, the probability of detection at $x_{0}$ is

$$
P\left(x_{0}\right)=\frac{n_{T}\left(x_{0}\right)}{\int n_{T}(x) d x} \approx \frac{\left|\Psi\left(x_{0}\right)\right|^{2} d V}{\int|\Psi(x)|^{2} d x}=\left|\Psi\left(x_{0}\right)\right|^{2} d V .
$$

Here $\Psi(x)$ is a kind of the wave function, a normalized vector of the $L_{2}$-space. (We state again that we still consider just classical signal theory.)

Conclusion. Born's rule is valid for probabilities of "discretized detection" of classical random signals under the following assumptions:

(a) ergodicity;

(b) a detector clicks after it "has eaten" approximately a portion of energy $\epsilon$;

(c) a detector is calibrated in such a way that this click threshold $\epsilon$ equals to the average energy of a signal (or more generally a class of signals);

(d) the energy is collected by this detector through time integration of signal's energy;

(e) the interval of integration $\Delta$ is long enough from the viewpoint of the internal time scale of a signal.

The assumption (e) is necessary to match (a). We remark that the internal time scale of a signal, i.e., the scale of its random fluctuations, has to be distinguished from the time scale of macroscopic measurement (observer's time scale). The former is essentially finer than the latter.

We presented a natural scheme of discrete detections which is based on time integration of signal's energy by a detector. Calibration of the detector plays a crucial role. This scheme applied to classical random signals reproduces Born's rule for discrete clicks. 


\section{Invariance of Born's rule with re- spect to calibration of detectors}

Let us repeat the previous considerations by scaling the detection threshold. We now put

$$
\epsilon=C\|\Psi\|^{2},
$$

where $C>0$ is an arbitrary scaling constant. We interpret scaling (33) as calibration of detector.

We now repeat considerations of section 3 with this scaled threshold. For example, the analog of the equality (28) has the form

$$
\frac{\Delta_{C}}{\gamma}\left|\psi\left(x_{0}\right)\right|^{2} d V \approx C\|\psi\|^{2}
$$

or for the normalized function $\Psi(x)=\psi(x) /\|\psi\|$,

$$
\frac{\Delta_{C}}{\gamma}\left|\Psi\left(x_{0}\right)\right|^{2} d V \approx C
$$

Thus at the point $x_{0}$ such a detector clicks (in average) with the frequency

$$
\lambda_{C}\left(x_{0}\right)=\frac{\gamma}{\Delta_{C}} \approx \frac{\left|\Psi\left(x_{0}\right)\right|^{2} d V}{C} .
$$

As in section 3, consider a large interval of time, say $T$. The number of clicks at $x_{0}$ during this interval is given by

$$
n_{T, C}\left(x_{0}\right)=\frac{T}{\Delta_{C}} \approx \frac{1}{\gamma C}\left|\Psi\left(x_{0}\right)\right|^{2} d V .
$$

Hence, the probability of detection at $x_{0}$ is

$$
P\left(x_{0}\right)=\frac{n_{T, C}\left(x_{0}\right)}{\int n_{T, C}(x) d x} \approx \frac{\left|\Psi\left(x_{0}\right)\right|^{2} d V}{\int|\Psi(x)|^{2} d x}=\left|\Psi\left(x_{0}\right)\right|^{2} d V .
$$

Surprisingly the probability of detection does not depend on calibration. (But the frequency of clicks decreases with increase of the scaling constant $C$. Hence, if one increases of the calibration level $C$, then more time will be needed to collect enough clicks to obtain a proper frequency estimation of the probability of detection.) 


\section{$5 \quad$ No double clicks}

We recall that Bohr elaborated his complementarity principle 3 from analysis of the two slit-experiment. On the one hand, quantum systems exhibit interference properties which are similar to properties of classical waves. On the other hand, these systems also exhibit particle properties. Wave properties (interference) are exhibited if both slits are open and experimenter does not try to control "which slit passing". At this experimental context one can be totally fine with a classical wave type model. However, if experimental context is changed and detectors are placed behind slits, then "wave features of quantum systems disappear and particle features are exhibited." What does the latter mean? Why is the usage of the wave picture impossible? Typically, it is claimed that, since classical wave is spatially extended, two detectors (behind both slits) can click simultaneoulsy and produce double clicks. However, as it is commonly claimed, there are no double clicks at all; hence, the wave model has to be rejected (in the context of the presence of detectors). Bohr had not find any reasonable explanation of context dependent features of quantum systems and he elaborated the complementarity principle.

Of course, the cliam that there are no double clicks at all is meaningless at the experimental level. There are always double clicks. The question is whether the number of double clicks is very small (comparing with the numbers of single clicks). Corresponding experiments have been done [19], [20] and it was shown that the number of double clicks is relatively small. Such experiments are considered as confirmation of Bohr's complementarity principle.

We show that the absence of double clicks might be not of the fundamental value, but a consequece of the procedure of calibration of detectors. Consider again a random signal $\phi$. But now we take two threshold type detectors located in neighbourhoods $V_{x_{0}}$ and $V_{y_{0}}$ of the points $x_{0}$ and $y_{0}$. They are calibrated with the connstant $C$, see (33). For moments of clicks, we have two approximate equalities:

$$
\begin{aligned}
& \frac{1}{\gamma} \int_{0}^{\Delta_{C}\left(x_{0}\right)} \int_{V_{x_{0}}}|\phi(s, x)|^{2} d x d s \approx C \epsilon, \\
& \frac{1}{\gamma} \int_{0}^{\Delta_{C}\left(y_{0}\right)} \int_{V_{y_{0}}}|\phi(s, x)|^{2} d x d s \approx C \epsilon,
\end{aligned}
$$

\footnotetext{
${ }^{3}$ This principle is often called "wave-particle" duality. However, we stress that Bohr had never used the latter terminology by himself.
} 
A double click corresponds to the (approximate) coincidence of moments of clicks

$$
\Delta_{C}\left(x_{0}, y_{0}\right)=\Delta_{C}\left(x_{0}\right)=\Delta_{C}\left(y_{0}\right) .
$$

Hence, by adding the approximate equalities (39), (40) under condition (41) we obtain

$$
\frac{1}{\gamma} \int_{0}^{\Delta_{C}\left(x_{0}, y_{0}\right)} \int_{V_{x_{0}} \cup V_{y_{0}}}|\phi(s, x)|^{2} d x d s \approx 2 C \epsilon,
$$

Again by using ergodicity and the assumption that the internal time scale of signals is essentially finer than the time scale of measurement ("click production") we obtain

$$
\begin{gathered}
\frac{\Delta_{C}\left(x_{0}, y_{0}\right)}{\gamma}\left[\frac{1}{\Delta_{C}\left(x_{0}, y_{0}\right)} \int_{0}^{\Delta_{C}\left(x_{0}, y_{0}\right)} \int_{V_{x_{0}} \cup V_{y_{0}}}|\phi(s, x)|^{2} d x d s\right] \\
\approx \frac{\Delta_{C}\left(x_{0}, y_{0}\right)}{\gamma} \int_{V_{x_{0}} \cup V_{y_{0}}}|\psi(x)|^{2} d x \approx 2 C\|\psi\|^{2}
\end{gathered}
$$

or, for normalized "wave function" $\Psi(x)$,

$$
\begin{aligned}
& \frac{\Delta_{C}\left(x_{0}, y_{0}\right)}{\gamma}\left[\int_{V_{x_{0}}}|\Psi(x)|^{2} d x+\int_{V_{y_{0}}}|\Psi(x)|^{2} d x\right] \\
= & \frac{\Delta_{C}\left(x_{0}, y_{0}\right)}{\gamma}\left[P\left(x \in V_{x_{0}}\right)+P\left(x \in V_{y_{0}}\right] \approx 2 C .\right.
\end{aligned}
$$

Hence

$P($ double click $)=\frac{\gamma}{\Delta_{\mathrm{C}}\left(\mathrm{x}_{0}, \mathrm{y}_{0}\right)} \approx \frac{1}{2 \mathrm{C}}\left[\mathrm{P}\left(\mathrm{x} \in \mathrm{V}_{\mathrm{x}_{0}}\right)+\mathrm{P}\left(\mathrm{x} \in \mathrm{V}_{\mathrm{y}_{0}}\right)\right] \leq \frac{1}{2 \mathrm{C}}$.

Hence, by increasing the calibration constant $C$ one is able to decrease the number of double clicks to negligibly small.

\section{Quantum probabilities from measure- ments of prequantum random fields}

As was discussed in introduction, in a series of papers [24]-[26] a purely wave model reproducing quantum averages was created - PCSFT (prequantum classical statistical field theory). The main problem in 
matching PCSFT with QM was violation of the spectral postulate of QM: prequantum physical varaibles are continuous quadratic forms of prequantum signals, but in the realistic measurement procedures we have discrete clicks of detectors. We shall see that the aforementioned detection scheme can be coupled to PCSFT and the problem of discrete clicks can be solved.

\subsection{Essentials of prequantum classical statis- tical field theory}

Take Hilbert space $H$ as space of classical states. Consider a probability distribution $\mu$ on $H$ having zero average (it means that

$$
\int_{H}\langle y, \phi\rangle d \mu(\phi)=0
$$

for any $y \in H$.) and covariance operator $D$ which is defined by a symmetric positively defined bilinear form:

$$
\left\langle D y_{1}, y_{2}\right\rangle=\int_{H}\left\langle y_{1}, \phi\right\rangle\left\langle\phi, y_{2}\right\rangle d \mu(\phi), y_{1}, y_{2} \in H,
$$

By scaling we obtain operator

$$
\rho=D / \operatorname{TrD}
$$

with $\operatorname{Tr} \rho=1$. In PCSFT it is considered as a density operator.

By PCSFT a quantum state, a density operator, is simply the symbolic representation of the covariance operator of the corresponding prequantum (classical) probability distribution - random field.

In general a probability distribution is not determined by its covariance operator. Thus the correspondence PCSFT $\rightarrow$ QM is not one-to-one. However, if the class of prequantum probability distributions is restricted to Gaussian, then this correspondence becomes one-to-one.

In PCSFT classical variables are defined as functions from (Hilbert) state space $H$ to real numbers, $f=f(\phi)$. By PCSFT a quantum observable, a self-adjoint operator, is simply a symbolic representation of $f$ by means of its second derivative (Hessian),

$$
f \rightarrow \widehat{A}=\frac{1}{2} f^{\prime \prime}(0)
$$


see [26] for detail. This correspondence is neither one-to-one. However, by restricting the class of classical variables to quadratic forms on Hilbert state space $H$,

$$
f_{A}(\phi)=\langle\widehat{A} \phi, \phi\rangle,
$$

we make correspondence PCSFT $\rightarrow$ QM one-to-one.

And finally, we present the basic equality coupling the prequantum (classical random field) and quantum (operator) averages

$$
\left\langle f_{A}\right\rangle_{\mu}\left(\equiv E f_{A}(\phi)\right)=\int_{M} f_{A}(\phi) d \mu(\phi)=\operatorname{TrD} \widehat{\mathrm{A}}=(\operatorname{TrD})\langle\widehat{\mathrm{A}}\rangle_{\rho} .
$$

Thus the quantum average $\langle\widehat{A}\rangle_{\rho}$ can be obtained as scaling of the classical average. We remark that scaling parameter $\operatorname{TrD}$ is, in fact, the dispersion of the probability distribution $\mu$ :

$$
\sigma^{2} \equiv E\|\phi\|^{2}=\int_{H}\|\phi\|^{2} d \mu(\phi)=\operatorname{Tr} D .
$$

It determines the scale of fluctuations of the prequantum random field.

\subsection{Born's rule from prequantum classical sta- tistical field theory}

An important class of quantum states is given by pure states, normalized vectors $\Psi \in H$. Any pure state $\Psi$ determines the density operator

$$
\rho=|\Psi\rangle\langle\Psi| \text {. }
$$

Take now a pure quantum state $\Psi$, i.e., $\|\Psi\|=1$. Consider a prequantum random field $\phi(x, \omega)$ (e.g., Gaussian) with zero average and the copvariance operator $D_{\psi}=|\psi\rangle\langle\psi|$, where

$$
\psi=\sqrt{\epsilon} \Psi \text {, }
$$

where $\epsilon>0$ is a small parameter such that $\epsilon^{2}$ has the physical dimension of energy density. (The wave function of QM has the physical dimension of 1 /volume.) Here $\epsilon=\int_{H}\|\phi\|^{2} d \mu_{\psi}(\phi)$ is the average energy of the prequantum random field. In the process of measurement this field is considered as a random signal.

Now we apply to this (so to say prequantum) random signal the scheme of section 3 and obtain the Born's rule.

Thus we presented a model of discrete detection of prequantum random fields corresponding to quantum systems which reproduces the basic rule fo $Q M$, the Born's rule. 


\section{$7 \quad$ The case of arbitrary covariance(density) operator}

We now generalize the above scheme of classical signal measurement to signals with arbitrary covariance operators. (We recall that we had considered only a very special class of signals with covariance operators given by one-dimensional projectors, see (15).) By applying this scheme to classical signals corresponding to quantum systems we obtain the Born's rule for quantum states given by density operators. So, let $\phi(x, \omega)$ be a random signal with the covariance operator $D_{\mu}$, where $\mu$ is the probability distribution of this random signal, a normalized measure on the $L_{2}$-space. Since this is a trace class operator it can be represented as an integral operator:

$$
D_{\mu} \phi(x)=\int_{\mathbf{R}^{3}} D_{\mu}(x, y) \phi(y) d y .
$$

Let us consider the integral operator with the kernel

$$
A_{x_{0}}(x, y)=\delta\left(x-x_{0}\right) \delta\left(y-x_{0}\right)
$$

where $x_{0} \in \mathbf{R}^{3}$ is a fixed point. Hence, $\widehat{A}_{x_{0}} \phi(x)=\phi\left(x_{0}\right) \delta\left(x-x_{0}\right)$ and

$$
\left\langle\widehat{A}_{x_{0}} \phi, \phi\right\rangle=\phi\left(x_{0}\right) \int \delta\left(x-x_{0}\right) \overline{\phi(x)} d x=\left|\phi\left(x_{0}\right)\right|^{2} .
$$

Set $\widehat{B}=D_{\mu} \widehat{A}_{x_{0}}$. Then it has the kernel

$$
B(u, v)=\int D_{\mu}(u, z) A_{x_{0}}(z, v) d z=D_{\mu}\left(u, x_{0}\right) \delta\left(v-x_{0}\right) .
$$

Hence

$$
\operatorname{Tr} \widehat{B}=\int \mathrm{B}(\mathrm{u}, \mathrm{u}) \mathrm{du}=\mathrm{D}_{\mu}\left(\mathrm{x}_{0}, \mathrm{x}_{0}\right)
$$

Thus

$$
\int_{H}\left|\phi\left(x_{0}\right)\right|^{2} d \mu(\phi)=\int_{H}\left\langle\widehat{A}_{x_{0}} \phi, \phi\right\rangle d \mu(\phi)=\operatorname{Tr}_{\mu} \widehat{\mathrm{A}}_{\mathrm{x}_{0}}=\mathrm{D}_{\mu}\left(\mathrm{x}_{0}, \mathrm{x}_{0}\right) .
$$

We also have

$$
\langle\pi\rangle=\operatorname{Tr} \mathrm{D}_{\mu}=\int_{\mathbf{R}^{3}} \mathrm{D}_{\mu}(\mathrm{x}, \mathrm{x}) \mathrm{dx} .
$$


We now generalize the detection scheme of section 3. We can directly jump to the generalization of the relation (28):

$$
\frac{\Delta}{\gamma} D_{\mu}\left(x_{0}, x_{0}\right) d V \approx \int_{\mathbf{R}^{3}} D_{\mu}(x, x) d x
$$

or

$$
\frac{\gamma}{\Delta} \approx \frac{D_{\mu}\left(x_{0}, x_{0}\right) d V}{\int_{\mathbf{R}^{3}} D_{\mu}(x, x) d x}
$$

Thus the probability of signal detection at the point $x_{0}$ is given by

$$
P\left(x_{0}\right) \approx \frac{D_{\mu}\left(x_{0}, x_{0}\right) d V}{\int_{\mathbf{R}^{3}} D_{\mu}(x, x) d x} .
$$

To find coupling with $\mathrm{QM}$, we set $\rho=D_{\mu} / \operatorname{Tr} \mathrm{D}_{\mu}$. Then $\operatorname{Tr} \rho=1$ and the above equality can be written in terms of the density operator $\rho:$

$$
P\left(x_{0}\right) \approx \rho\left(x_{0}, x_{0}\right) d V .
$$

This is nothing else than the Born's rule for the quantum state given by the density operator $\rho$. Thus we demosntrated that even for a mixed quantum state it is possible to find such a prequantum random field that the "discrete-click" measurement of this signal produces the basic probabilistic rule of QM.

\section{Discrete-counts detection of classi- cal random signals: general scheme}

Let $e(x)$ be a fixed $L_{2}$-function. We present a version of the discretedetection scheme of section 3 by taking $e(x)$, instead of $\delta_{x_{0}}(x)=\delta(x-$ $x_{0}$ ). (In principle, we may proceed without the restriction that $e \in L_{2}$ and select $e$ as a distribution; but we proceed with $L_{2}$-functions; on one hand this simplifies essentially mathematics of presentation; on the other hand, in this section we are mainly interested in a signaldetection scheme underlying quantum measurements of observers with discrete spectra.) Consider a detector, "e-antenna", corresponding to the function $e(x)$. It works in the following way. As in the scheme of section 3, it integrates the energy of a signal during some interval of time, until the thershold $\epsilon$ is approached. Here $\epsilon$ is the average energy of a random signal. The only difference with section 3 is that such a detector integrates the "energy along the direction" $e \in L_{2}$, namely, 
$\frac{1}{\gamma} \int_{0}^{\Delta}|\langle\phi(s, x), e\rangle|^{2} d s$. The rest of the detection scheme coincides with the one presented section 3 (see also section 7 ):

$$
\frac{1}{\gamma} \int_{0}^{\Delta}|\langle\phi(s, x), e\rangle|^{2} d s \approx \epsilon=\operatorname{TrD}_{\mu}
$$

where $D_{\mu}$ is the covarince operator of the probability distribution of a random signal under measurement.

$$
\begin{gathered}
\frac{\Delta}{\gamma}\left(\frac{1}{\Delta} \int_{0}^{\Delta}|\langle\phi(s, x), e\rangle|^{2} d s\right) \approx \operatorname{TrD}_{\mu}, \\
\frac{\Delta}{\gamma} \int_{H}|\langle\phi, e\rangle|^{2} d \mu(\phi) \approx \operatorname{TrD}_{\mu}, \\
\frac{\Delta}{\gamma}\left\langle D_{\mu} e, e\right\rangle \approx \operatorname{TrD}_{\mu},
\end{gathered}
$$

finally, for the probability of detection of this signal by the $e$-antenna, we get

$$
P(e) \approx \frac{\left\langle D_{\mu} e, e\right\rangle}{\operatorname{TrD}_{\mu}} .
$$

Now to proceed to QM, we first normalize the covariance operator of the random signal, $\rho=D_{\mu} / \operatorname{Tr} \mathrm{D}_{\mu}$. Hence,

$$
P(e) \approx\langle\rho e, e\rangle \text {. }
$$

Consider now a quantum observable $\widehat{A}$ with purely discrete nondegenerate spectrum; $\widehat{A} e_{k}=\lambda_{k} e_{k}$. Consider the ensemble of detectors corresponding to the system of normalized eigenfunctions $\left\{e_{k}(x)\right\}$; ensemble of $e_{k}$-antennas. Each click induced by the $e_{k}$-antenna is associated with the value $\lambda_{k}$. Denote this classical observable by the symbol $M_{A}$. The average of $M_{A}$ is given by

$$
\left\langle M_{A}\right\rangle=\sum_{k} \lambda_{k} P\left(e_{k}\right)=\sum_{k} \lambda_{k}\left\langle\rho e_{k}, e_{k}\right\rangle .
$$

Thus we obtained the quantum formula for averages:

$$
\left\langle M_{A}\right\rangle=\operatorname{Tr} \rho \widehat{\mathrm{A}} .
$$

We now can extend our approach (representation of quantum measurements as "click-measurements" of classical random signals) to quantum observables with discrete degenerate spectra. Consider a 
subspace $L$ of $H$ and the corresponding $L$-antenna. Let $\widehat{P}_{L}$ be the corresponding orthogonal projector. The $L$-antenna produces a click if

$$
\begin{gathered}
\frac{1}{\gamma} \int_{0}^{\Delta}\left\|\widehat{P}_{L} \phi(s)\right\|^{2} d s \approx \epsilon=\operatorname{TrD}_{\mu} \\
\frac{\gamma}{\Delta} \approx \operatorname{TrD}_{\mu} \widehat{\mathrm{P}}_{\mathrm{L}} / \operatorname{TrD}_{\mu}
\end{gathered}
$$

i.e.,

$$
P(L) \approx \operatorname{TrD}_{\mu} \widehat{\mathrm{P}}_{\mathrm{L}} / \operatorname{TrD}_{\mu},
$$

where $\rho$ is the normalization of the covariance operator of the signal.

Now take a quantum observable $\widehat{A}$ with discrete in general degenerate spectrum; consider its eigenspaces $L_{k}$ corresponding eigenvalues $\lambda_{k}$ Then measurements of this quantum observable can be modeled through measurements performed by a system of $L_{k}$-antennas.

In this approach there is no reason to consider orthogonal projectors and self-adjoint operators; a POVM, $\left\{\widehat{Q}_{k}\right\}, \sum_{k} \widehat{Q}_{k}=I, \widehat{Q}_{k} \geq 0$, arises naturally as the symbolic representation of generalization of aforementioned scheme (of measurement of classical random signals) to the family of $Q_{k}$-antennas: each integrates the $Q_{k}$-transform of a classical signal. The starting point of generalization is the realtion:

$$
\frac{1}{\gamma} \int_{0}^{\Delta}\left\|\widehat{Q}_{k} \phi(s)\right\|^{2} d s \approx \epsilon=\operatorname{TrD}_{\mu}
$$

By starting with Dirac's approach to quantum observables (based on generalized eigenvectors) we can easily generalize our scheme to operators with continuous spectra and, in particular, obtain the position measurement as a special case.

This paper was written under support of the grant QBIC (20082011), Tokyo University of Science, and the grant Mathematical Modeling, Linnaeus Unievrity (2008-2010).

\section{References}

[1] A. Einstein, B. Podolsky, N. Rosen, Phys. Rev. 47, 777 (1935)

[2] A. Plotnitsky, J. Modern Optics 54, 2393 (2007)

[3] A. Plotnitsky, Epistemology and Probability: Bohr, Heisenberg, Schrdinger, and the Nature of Quantum-Theoretical Thinking. (Springer, Berlin-New York, 2009). 
[4] L. Accardi, G. Adenier, C. A. Fuchs, G. Jaeger, A. Khrennikov, J.-A. Larsson, S. Stenholm (eds.), Foundations of Probability and Physics-5, American Institute of Physics, Ser. Conference Proceedings 1101, Melville, NY (2009)

[5] G. Adenier, A. Khrennikov, and Th.M. Nieuwenhuizen (eds.), Quantum Theory: Reconsideration of Foundations-3. American Institute of Physics, Ser. Conference Proceedings 810, Melville, NY (2006)

[6] G. GAdenier, C. Fuchs, and A. Khrennikov (eds.),Foundations of Probability and Physics-3. American Institute of Physics, Ser. Conference Proceedings 889, Melville, NY (2007)

[7] G. Adenier, A. Khrennikov, P. Lahti, V. I. Manko, and Th.M. Nieuwenhuizen (eds.), Quantum theory: reconsideration of foundations-4, American Institute of Physics, Ser. Conference Proceedings 962, Melville, NY (2008)

[8] G. Jaeger, Entanglement, Information, and the Interpretation of Quantum Mechanics (The Frontiers Collection). (BerlinHeidelberg-New York, Springer, 2010)

[9] G. Jaeger, Quantum Information: An Overview. (BerlinHeidelberg-New York, Springer, 2010)

[10] P. Pearle, Phys. Rev. D 2, 1418 (1970)

[11] N. Gisin and B. Gisin, Phys. Lett. A. 260, 323 (1999)

[12] J.-A. Larsson, Quantum Paradoxes, Probability Theory, and Change of Ensemble. (Linköping Univ. Press, Linköping, 2000)

[13] G. Adenier, American Journal of Physics 76, 147 (2008)

[14] G. Adenier and A. Khrennikov, Phys. B: Atomic, Molecular and Optical Physics 40 (1), 131 (2007)

[15] A. Aspect, Three experimental tests of Bell inequalities by the measurement of polarization correlations between photons, $\mathrm{PhD}$ Thesis 2674( Orsay), 1983 (In French).

[16] A. Aspect, J. Dalibard, and G. Roger, Phys. Rev. Lett. 49, 91 (1982)

[17] G. Weihs, An experiment to test Bells inequality under Einstein locality. PhD Thesis, University of Innsbruck, 1999 (In German).

[18] G. Weihs, Th. Jennewein, Ch. Simon, H. Weinfurter, and A. Zeilinger, Phys. Rev. Lett. 81(23), 5039 (1998) 
[19] P. Grangier, G. Roger, and A. Aspect, EPL 1, 173 (1986)

[20] P. Grangier, Etude expérimentale de propriétés non-classiques de la lumière: interférence à un seul photon. Université de Paris-Sud, Centre D’Orsay (1986)

[21] G. F. Knoll, Radiation, Detection and Measurement. (New York, John Wiley and Sons, 1979, 2000)

[22] W. D. Oliver, E. Diamanti, E. Waks, K. Inoue, and Y. Yamamoto, Selected Topics in Quant. Electronics 9(6), 15022003

[23] S. V. Polyakov, A. Migdall, and S. W. Nam, Real-time dataacquisition platform for pulsed measurements. In: Advances in Quantum Theory, pp. 505-519, American Institute of Physics, Ser. Conference Proceedings 1327, Melville, NY (2011)

[24] A. Khrennikov, J. Phys. A: Math. Gen. 38, 9051 (2005; Found. Phys. Letters 18, 637 (2006); Physics Letters A 357, 171 (2006); Found. Phys. Lett. 19, 299 (2006); Nuovo Cimento B 121, 505 (2006)

[25] A. Khrennikov, EPL 88, 40005.1 (2009); Europhysics Lett. 90, art-number 40004 (2010); J. of Russian Laser Research, 31 (2), 191(2010)

[26] A. Khrennikov, Nuovo Cimento B 121 (9), 1005 (2006)

[27] A. Khrennikov, M. Ohya, and N. Watanabe, J. Russian Laser Research 31, 462(2010)

[28] L. de Broglie, Recherches sur la thorie des quanta (Researches on the quantum theory), Thesis, Paris, 1924.

[29] L. de Broglie, Non-linear Wave Mechanics: A Causal Interpretation. (Amsterdam: Elsevier, 1960)

[30] E. Schrödinger, Naturwissenschaften 23, 807; 823; 844 (1935)

[31] E. Schrödinger, E. Schrödinger Gesammelte Abhandlungen. Wieweg and Son, Wien (1984); especially the paper "What is an elementary particle?", pp. 456-463.

[32] A. Einstein and L. Infeld, Evolution of Physics: The Growth of Ideas from Early Concepts to Relativity and Quanta. (Simon and Schuster, 1961)

[33] W. E. Lamb and M. O. Scully, The photoelectric effect without photons. In: Polarization, Matter and Radiation. Jubilee volume 
in honour of Alfred Kasiler, pp.363-369. Press of University de France, Paris (1969)

[34] W. E. Lamb, The Interpretation of Quantum Mechanics. (Edited and annotated by Mehra, Ja.) (Rinton Press, Inc., Princeton, 2001)

[35] A. Lande, Foundations of Quantum Theory. (Yale Univ. Press, 1955)

[36] A. Lande, New Foundations of Quantum Mechanics. (Cambridge Univ. Press, Cambridge, 1965)

[37] D. Bohm and B. Hiley, The Undivided Universe: an Ontological Interpretation of Quantum Mechanics. (Routledge and Kegan Paul, London, 1993)

[38] H. H. Louisell, Quantum Statistical Properties of Radiation. (New York, J. Wiley, 1973)

[39] L. Mandel and E. Wolf, Optical Coherence and Quantum Optics. (Cambridge, Cambridge University Press, 1995)

[40] M.O. Scully and M.S. Zubairy, Quantum Optics. (Cambridge: Cambridge University Press, 1997)

[41] L. De la Pena and A. Cetto, The Quantum Dice: An Introduction to Stochastic Electrodynamics. (Dordrecht, Kluwer, 1996)

[42] A. Casado, T. Marshall, E. Santos, J. Opt. Soc. Am. B 14, 494 (1997)

[43] G. Brida, M. Genovese, M. Gramegna, C. Novero, and E. Predazzi, Phys. Lett A 299, 121 (2002)

[44] T. Boyer, A brief survey of stochastic electrodynamics. Foundations of Radiation Theory and Quantum Electrodynamics ed Barut A (New York: Plenum), p. 141 (1980)

[45] D. C. Cole, A. Rueda, K. Danley, Phys. Rev. A 63054101 (2001)

[46] Th. Nieuwenhuizen, Classical phase space density for relativistic electron Quantum Theory: Reconsideration of Foundations-3 (Ser. Conference Proceedings vol 810) (Melville, NY: American Institute of Physics) p. 198 (2006)

[47] S. M. Ritov, Introduction to Statistical Radiophysics. (Nauka, Fizmatlit, Moscow, 1966). 
[48] P. M. Morse and K. U. Ingard, Theoretical Acoustics. (Mc GrawHill Book Company, New York, St. Louis, San Francisco, Toronto, London, Sydney, 1968)

[49] M. Ozawa, Lecture Notes in Math. 1021, 518 (1983)

[50] M. Ozawa, Rep. Math. Phys. 18, 11 (1980)

[51] G. M. D'Ariano and A. Tosini, Quant. Inf. Proc. 9, 95(2010)

[52] G, M. D'Ariano, Probabilistic Theories: What is Special about Quantum Mechanics? in Philosophy of Quantum Information and Entanglement, Eds A. Bokulich and G. Jaeger (Cambridge University Press, Cambridge, p. 85 (2010)

[53] U. Sinha, C. Couteau, Z. Medendorp, I. Sllner, R. Laflamme, R. Sorkin, and G. Weihs, Testing Born's rule in quantum mechanics with a triple slit experiment. In: Accardi, L., Adenier, G., Fuchs, C., Jaeger, G., Khrennikov, A., Larsson, J.-A., and Stenholm, S. (eds). Foundations of Probability and Physics-5, , pp. 200-207. American Institute of Physics, Ser. Conference Proceedings, vol. 1101. Melville, NY (2009)

[54] U. Sinha, Ch. Couteau, Th. Jennewein, R. Laflamme, and G. Weihs, Science, 418, 23 July 2010.

[55] C. Ududec, H. Barnum, J. Emerson Three Slit Experiments and the Structure of Quantum Theory, arXiv:0909.4787.

[56] A. Khrennikov, Physica E: Low-dimensional Systems and Nanostructures 42, 287(2010) 